\title{
MeerTRAP: Real Time Commensal Searching for Transients and Pulsars with MeerKAT
}

\author{
Ben Stappers* \\ Jodrell Bank Centre for Astrophysics, School of Physics and Astronomy, University of \\ Manchester \\ E-mail: Ben.Stappers@manchester.ac.uk
}

\begin{abstract}
MeerTRAP: Meer (more) TRAnsients and Pulsars, is a project to commensally use the MeerKAT telescope to search the radio sky for pulsars and fast transients and to rapidly and accurately locate them. Utilising the excellent sensitivity and sky coverage of MeerKAT MeerTRAP will enable the discovery of many rare and scientifically important pulsar types: relativistic binaries, intermittent emitters, transitioning systems. Current radio telescopes have only explored the tip of the transients "iceberg" and MeerTRAP will transform our knowledge of these manifestations of extreme physics. It will detect hundreds of new bursts, which will all be well localised, allowing us to identify hosts and distances, greatly enhancing their use as cosmological probes. Localisation also enables measurement of their true fluxes, polarisation and spectral indices which are all crucial to identify their origin. It will do this by expanding the tied-array beam-forming capabilities provided by the MPIfR and add the capability to search the coherent and incoherent beams for transients and pulsars in real time. It will also implement a tied-array buffer on each of the dishes and the ability to use this data to image the field around bursts. It will also allow the extraction of high time resolution and polarisation calibrated data on each burst. MeerTRAP will also work with the MeerLICHT telescope to obtain optical data before, during and after the bursts. MeerTRAP is funded through a European Research Council Advanced Grant. The system will be designed in close collaboration with colleagues from the MPIfR and the University of Oxford and builds on the beam-former developed by the MPIfR. Descriptions of the beam-former and the S-band system developed by MPIfR and the related MeerKAT project TRAPUM can be found elsewhere in these proceedings.
\end{abstract}

MeerKAT Science: On the Pathway to the SKA

25-27 May, 2016

Stellenbosch, South Africa

${ }^{*}$ Speaker. 


\section{Introduction}

We can advance the frontiers of physics by observations of the extremes. Fast radio transients, with timescales typically less than a second, are likely associated with the extremes of energy, magnetic field strength, gravity and density. These sources provide a unique laboratory with which to probe physical regimes well beyond those achievable in any terrestrial experiment. Located at extragalactic distances, they can be used as probes of the content of the intergalactic medium (IGM) and probe the nature of the missing baryons. Moreover if they can be shown to be standard candles then they will be useful additional components of the distance ladder reaching out to redshifts up to 2 and perhaps beyond. We are only just scratching the surface in terms of our understandng of the fast radio transient sky, and so we are also exploring the unknown.

Detecting fast radio transients is extremely challenging; their sporadic nature requires either vast amounts of observing time and/or wider fields-of-view (FoV) to catch them. The known sources already span a range of durations from nanoseconds, e.g. giant pulses from the Crab pulsar, to seconds, e.g the Galactic centre transient GCRT J1745-3009 (see Figure 1). Likewise the luminosity spans 20 orders of magnitude, from Jupiter emission to the FRBs, however, the majority of the intervening parameter space is unexplored. Other known and proposed sources include: ultra-high-energy particles, annihilating black holes, merging neutron stars, supernovae and gamma-ray bursts, flare stars, planets (Solar \& Extrasolar), neutron stars (intermittent pulsars/magnetars/RRATs), extra-terrestrial intelligence and things that have not yet even been thought of! The fast transient phase space we are sensitive to with MeerTRAP is shown in Figure 1 and given the space restrictions here, we only discuss the fast radio bursts in detail below.

Radio pulsars are rapidly rotating neutron stars that emit radio emission from their magnetic poles. As the poles sweep across our radio telescope we detect "pulses" of emission much like the ticks of a clock. On long timescales the stability of these "clocks" rivals the precision of the atomic clocks on Earth and furnishes us with tools with which to study the extremes of energy, density, temperature and gravitational and magnetic fields. With MeerTRAP it will be possible discover many new pulsars but in particular to expand the range of sources known by using the unique MeerTRAP observing approach to discover the more "variable" systems as discussed below.

\section{Fast Radio Bursts}

The discovery of four new fast radio bursts (FRBs) confirmed that there is a population of millisecond duration bursts that are located at extragalactic distances [1]. Since the original detection [2], up to a dozen more have been detected at Parkes (e.g. [3]) the Arecibo telescope [4], the GBT [5] and UTMOST [6]. This population of bright ( $>1 \mathrm{Jy} \mathrm{ms}$ ) bursts exhibit quadratic frequency dependent delays in the arrival time of their pulses that places them at redshifts out to at least 1.5. Their extreme luminosity and intrinsic short duration suggests they are associated with extreme events such as supernovae and gamma-ray bursts. However their estimated rates may favour giant flares from soft gamma ray repeaters (e.g [7]). Many new mechanisms are being proposed ranging from the collapse of a supra-massive rotating neutron star to a black hole [8] to merging neutron stars [9]. One suggestion is that FRBs are supergiant pulsar pulses [10] and thus should repeat. Recently the Arecibo burst was seen to repeat [11] but none of the others have, to very good limits, 
and there is consideration that there might be more than one source of FRBs. The large number of FRBs discovered by MeerTRAP and repeat visits to the same field will probe an unmatched range of possible repeat timescales and provide crucial new information. More FRBs are being discovered and advances in instrumentation have enabled triggering of multi-wavelength follow up quite rapidly after the burst [12], and the recent detection of a possible afterglow and host galaxy [13] and subsequent discussion about the validity of the association highlight the value of being able to use the burst itself to localise the transient. With the transient buffers and MeerTRAP this will be possible, and the real time trigger will also allow for more rapid imaging possibilities to search for afterglows as well. Polarisation measurements have now also been possible, indicating either an unusual emission mechanism or severe linear depolarisation [12, 5].

The detection of fast, dispersed radio pulses from events at cosmological distances provides a tool to measure the nature of the magneto-ioinic intergalactic medium. More than half of all baryons have not been detected at $\mathrm{z}<2$ and most of these "missing" baryons may reside at temperatures and densities that are difficult to detect with current methods [14] but can be probed with dispersed bursts. Determining the dispersion measures for hundreds of FRBs allows the spatial distribution, and thus the origin of these baryons, to be resolved (e.g. [15]). A stacking anaylsis of about 100 FRBs, with arcminute localisations, could be used to study the baryonic mass profile surrounding different galaxy types, an important parameter in galaxy formation and feedback models [16]. Larger samples of FRBs, which have associated redshift information, have been proposed to constrain the dark energy equation of state [17].

It is important to note that our basic understanding of the measured properties of the known FRBs and their use as cosmological probes is significantly hampered by a lack of an accurate position for the burst location. MeerTRAP will be able to provide the required positional accuracy. While this discussion has concentrated on the case of FRBs any extragalactic population of sufficiently short duration dispersed radio transients discovered by MeerTRAP will deliver the same science. Moreover, as discussed above, the range of possible fast radio transient parameter space has barely been probed. MeerTRAP with its wide range of cadences, observing frequencies, sky locations and most importantly sensitivity and instantaneous FoV can greatly extend our knowledge of this parameter space (see Figure 1).

Another extremely exciting possibility is finding the electromagnetic counterpart to a gravitational wave burst either by providing triggers to, or receiving triggers from the Advanced LIGO and VIRGO detectors. This will significantly improve localisation of the gravitational wave source, helping to identify the progenitor and probe its environment and attributes. If the emission is associated with a standard siren, that is, a merging compact object, it could allow tests of gravity and measurement of the distance scale [18].

\section{Pulsars}

Relativistic binaries containing pulsars can be used for important physical tests and the more extreme the binary the better. Thus a pulsar in a compact and eccentric orbit with another compact object like a white dwarf, another neutron star, or a black hole is highly desired. These objects allow us to perform important tests of gravity theories: the strong equivalence principle, gravitational radiation and the "cosmic censorship conjecture" to name just a few (e.g. [19]). However, the 


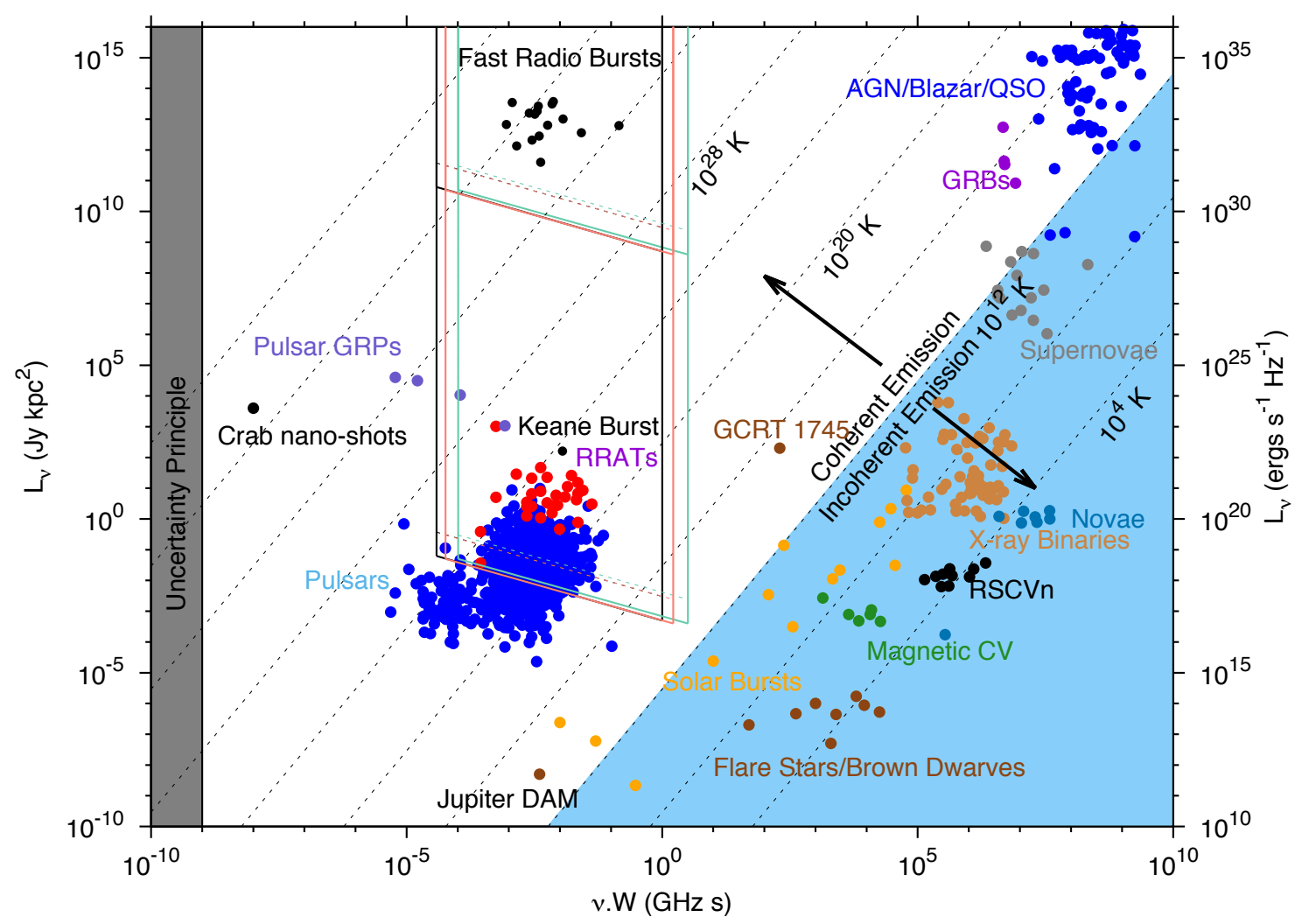

Figure 1: Transient parameter space showing specific luminosity against the product of observing frequency and transient duration. The boxes correspond to the 1-sigma sensitivity curves for MeerTRAP for source distances of $1 \mathrm{kpc}$ (lower curves) and $1 \mathrm{Gpc}$ (upper curves) and coherent (solid) and incoherent (dashed) modes. The different colours correspond to the different frequency bands: UHF (black), L-band (salmon) and Mid (cyan). One can see that all the known FRBs could be easily detected with MeerTRAP. Also the majority of known pulsars would be detected via their single pulses as would all RRATs. Credit Keane (after Cordes).

binary motion of the pulsar causes its rotation frequency to be significantly Doppler shifted making it extremely difficult to discover. Our MeerTRAP approach makes many visits to the same pieces of sky thus catching systems when the effect of binary motion is least, greatly improving our chances of discovering extreme binaries. Transition objects are neutron stars recently found to transition between radio emitting binary millisecond pulsar (MSP) and Low Mass X-ray Binary phases where radio pulsations cease (e.g. [20]). These systems provide vital clues to the mass outflow, accretion physics and emission processes involving neutron stars. They also tell us about the birth periods and masses of MSPs and thus have important consequences for the neutron star equation of state. The short timescale for which they are radio emitters and their relatively low X-ray luminosity make frequent pulsar search observations of the same areas of sky the ideal way to catch them as they turn on as radio emitters.

RRATs are rotating neutron stars that emit extremely infrequently, less than a second per day [21]. If they are distinct from pulsars then there may be too many neutron stars in the Galaxy compared to what is expected from the supernova rate [22]. A much larger sample, with measured 
ages, magnetic field strengths and accurate positions, is required. The discovery of RRATs will benefit greatly from the wide field of view (FoV) and regular visits to the same regions possible with MeerTRAP. Accurate measurement of their position will be possible with the transient buffer we propose here, greatly aiding the determination of the spin parameters. Intermittent pulsars are a pulsar class that emit for up to hundreds of days and then turn off completely for up to hundreds of days [23]. These switches are accompanied by changes in the rate at which the pulsar spins down: indicating significant changes in the pulsar magnetosphere. At present only a few systems are known, as the long periods of radio silence mean they are very difficult to detect. More sources will provide understanding of the neutron star population size, the radio emission-spin properties connection and relation to other radio variability like nulling and moding. The minutes to months cadences of MeerTRAP give an unbiased range of timescales to detect these sources.

\section{Goals of MeerTRAP}

We can summarise the goals of MeerTRAP as being:

Goal 1 The bursting sky: Detect hundreds of Galactic and extragalactic short duration radio bursts from extreme and cataclysmic environments to understand the associated physics. Identify prompt radio emission potentially associated with events like gamma-ray bursts and gravitational wave emission. Determine accurate positions of the fast radio bursts (FRBs) allowing us to distinguish formation models and use them as cosmological probes through their interaction with the intergalactic medium and space-time.

Goal 2 The periodic sky: Discover radio pulsars over an unprecedented range of parameter space by covering: variability on timescales from days to years, luminosity, spectral index, binarity and multiple systems. This will give us the most comprehensive view yet of the radio emitting neutron star population with the implications this has for star formation history, binary evolution and neutron star merger rates. It will reveal individual systems that can be used to test gravity theories, probe the equation of state of ultra-dense matter and enable the direct detection of nano-hertz gravitational waves.

\section{What is MeerTRAP?}

MeerTRAP is a project funded by the European Research council through an Advanced Grant. This funding pays for the hardware and software, described below, to enable the capability required to achieve the MeerTRAP science goals, but also funds postdoctoral researchers, $\mathrm{PhD}$ students and other staff to deliver the requisite software and to undertake the cutting edge science. MeerTRAP will utilise a fully commensal observing strategy, it will undertake piggyback observations on all of the agreed MeerkAT Large Survey Projects (MLSPs) time (as already agreed with all the MLSP PIs) and where possible it will also utilise time when the antennas are stowed. This will result in the largest ever total observing time for a sensitive pulsar and fast transient survey. To achieve this we will implement a unique capability by extending a 200-beam beamformer being supplied by the Max Planck Institute for Radio Astronomy to 400 beams and outfitting it with transient 


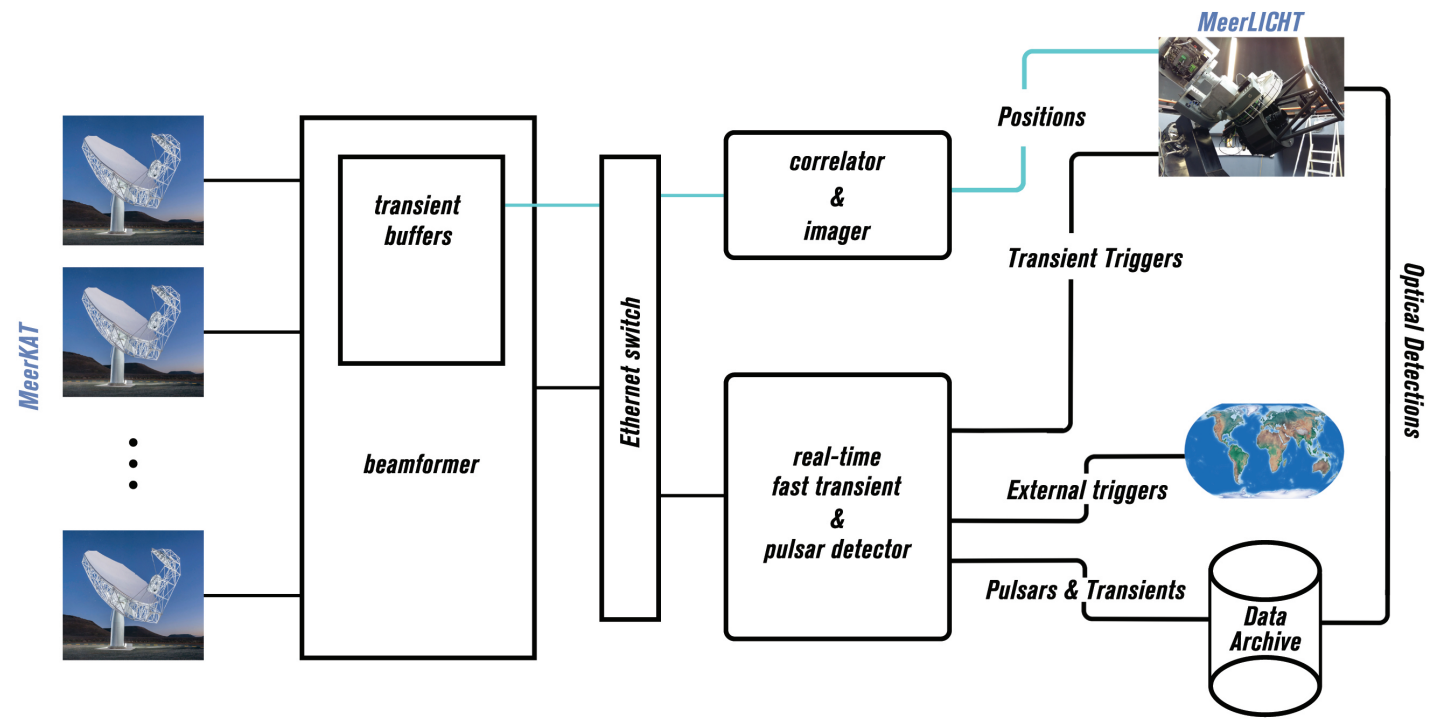

Figure 2: A functional diagram of MeerTRAP indicating the components and functions that will be implemented. Black lines show the transient and pulsar detection processing path and the blue line indicates the localisation and generation of triggers and the optical observations with MeerLICHT.

buffers. We will also add the capability (hardware and software) to extend their offline (non-real time) system to search for pulsars and transients over all the beams, all the time and in real time. This hardware will also perform correlations to make rapid images of the sky from the transient buffer data when triggered for possible transients. An overview of the different components of MeerTRAP are shown in Figure 2.

We will implement a coherent (in phase) and incoherent (adding intensities) mode (see Figure 3 for an example) for pulsar and transient searches. The coherent mode will combine the core dishes to achieve maximum sensitivity. The separation of the dishes results in a coherent beam with a small FoV, however we will use 400 beams for a total FoV of 0.1 square degrees. The incoherent sum of the 64 dishes results in a lower sensitivity but samples the entire 1.27 square degree FoV of a MeerKAT dish. It is essential that the search for dispersed fast radio transients take place in real time to enable rapid follow-up observations. We will use our coherent beam localisation method [24] to give (sub)arcsecond positions within a few seconds for bright transients. The detection of a burst will also trigger our transient buffers which will capture up to 30s of data, allowing the localisation of transients detected with dispersion measure of at least $3000 \mathrm{pc} \mathrm{cm}^{-3}$ (redshifts beyond 2). This buffer includes enough time for processing and triggering. The full bandwidth from all 64 dishes will be captured and imaged shortly after the trigger. The longer full-array baselines and improved sensitivity (compared to either the 40 dishes in the core or the incoherent sum) will allow for (sub)arcsecond localisation of transients detected in either the coherent or incoherent beams providing the precision to identify host galaxies for radio bursts at cosmological distances. It also allows us to trigger on much weaker bursts as the improved sensitivity allows us to confirm if they are real.

We have also joined the MeerLICHT project: an optical telescope with a 65-cm aperture, a 
FoV of 2.7 square degrees matched to the MeerKAT FoV. It will continuously point at the same location of the sky as MeerKAT giving simultaneous optical and radio data. MeerLICHT is in its final design phase and will soon be operational. Access to MeerLICHT data will allow us to search for prompt and afterglow emission, mine the rich multi-wavelength data available for many MLSP fields, activate proposals to get redshifts and high-energy data and to send out MeerTRAP triggers to the community maximising scientific return.

The main aim of searching for pulsars with MeerTRAP is to utilise the many multiple visits of varying cadence to the same regions of sky of the MLSPs. This will reveal pulsars where the signal varies in either intensity or period. The large number of beams and the continuous observing means that a real time pulsar search pipeline is the only option. For the pulsar search we will use a sampling time of $64 \mu \mathrm{s}$ and a channel bandwidth of $0.18 \mathrm{MHz}$ which gives us excellent sensitivity to MSPs, even up to dispersion measures of $1000 \mathrm{pc} \mathrm{cm}-3$ when using the mid-frequency band. Significant advances in using GPUs for pulsar searching and our SKA design and prototyping experience show that, for the modest acceleration range we will search, real time processing of all these dispersion measures is possible with the late-2017 era GPUs we plan to use. All the candidates will pass through an automated classifier (e.g. [25, 26]) and associated data will be archived and used when comparing results for future observations of the same region.

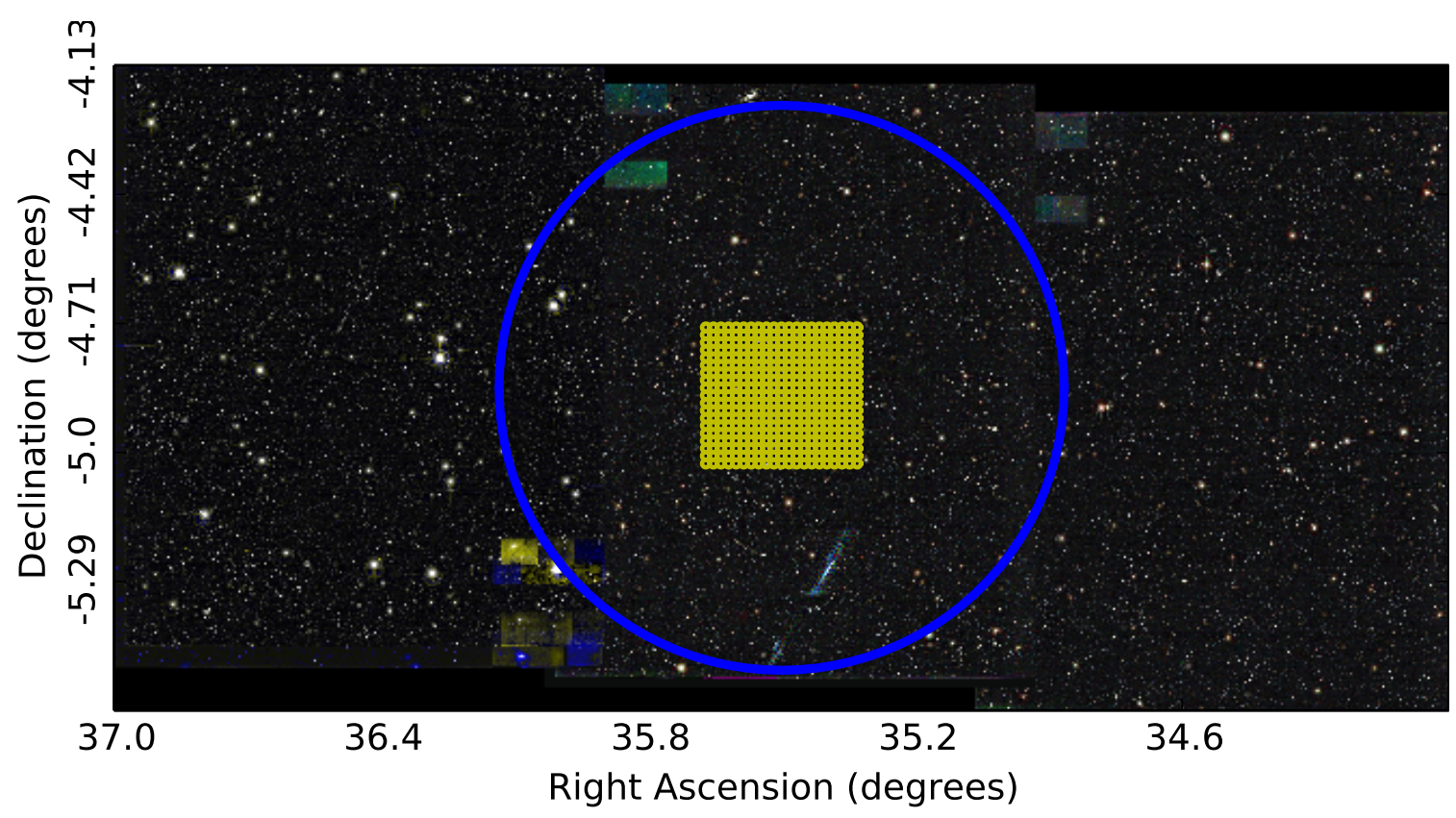

Figure 3: A 4.5 square degree part of the XMMLSS field that will be observed by MeerKAT Large Survey Project MIGHTEE. Overlaid is the FoV seen by the incoherent mode beam (blue) and the 400 coherent mode beams (yellow) of MeerTRAP. This shows the wide fields of view accessible to MeerTRAP, the value of piggybacking and the large supporting set of data that will exist. Note that the blurred regions correspond to bad chips on the optical camera. From VIDEO survey (Jarvis et al. 2013). Credit: Boris Hauessler

MeerTRAP will also help facilitate the goals of TRAPUM (TRansients and PUlsars with MeerKAT) which is an MLSP which is discussed in detail elsewhere in these proceedings, to search for pulsars and fast transients. It was noted during the original feedback to the MLSP process that if funding could be found to support the development of a beamformer and processing capability 
then it could be used to enhance the work that the MLSP could do. Prompted by this both the MPIfR and the University of Manchester pursued funding to be able to achieve this, and this has been successful with both the MPIfR beam former project and MeerTRAP. As part of enabling and distinguishing these projects it has been agreed with the TRAPUM team that the funding granted to the MPIfR and MeerTRAP will provide more capacity for the allocated MLSP time to TRAPUM. This means that the data obtained using this combined capacity in TRAPUM time will be available to the entire TRAPUM team. This will give TRAPUM access to a much greater field-of-view for searching for pulsars and fast transients and this is reflected in the updated TRAPUM science case (see these proceedings). The rest of the time when MeerKAT is doing MLSP observing MeerTRAP will be used to pursue MeerTRAP science primarily led by members of the MeerTRAP team.

\section{What will MeerTRAP find?}

With the number of FRBs known still small and thus the rates and luminosity distributions are poorly constrained and so estimates here are based on the current best numbers. The combination of sensitivity, FoV and observing time for MeerTRAP means that it is one of the best instruments to undertake these searches, while the fully commensal nature gives the requisite large fraction of on sky time. In incoherent mode our calculations shows that its wide FoV results in at least 200 new FRBs in 5 years. The smaller-FoV coherent mode reaches lower fluxes and will find about 100 FRBs, sufficient to determine their origin and start intergalactic medium and cosmological studies. These two MeerTRAP modes probe different flux limits and can constrain the luminosity function. While it may not find as many bursts, compared to instruments like CHIME and UTMOST, MeerTRAP has the advantage of having longer baselines (especially when we include the outermost dishes which we can with the transient buffers) and so will be able to provide very precise localisations well constrained in both right ascension and declination. VFASTR (VLBA) could provide precise localisation but it will discover less bursts. Surveys like those planned with the VLA have similar goals to those planned here but will use different detection methodologies. The advantages that the interferometers mentioned above have compared to the single dish experiments is that one can localise on the burst itself and thereby alleviate the need to identify an afterglow. Moreover, the accurate position makes it easier to identify any potential afterglow, or host, even if there is no emission at other wavelengths. Using a radio burst as a trigger can be a problem for the detection of prompt emission at other wavelengths as dispersion means there can be a significant time delay between when the burst occurred and when the radio emission was detected. With MeerTRAP we will circumvent this in the optical by working closely with the MeerLICHT telescope which will always be observing the same field as MeerKAT, allowing us to effectively look back in time to see if there was prompt optical emission.

The planned incoherent pulsar survey (MeerTRAP-I) has sensitivity comparable to the highly successful Parkes (HTRU) surveys, but has a larger FoV and through the commensal nature has a larger total observing time. The coherent survey (MeerTRAP-C) is extremely sensitive, comparable to Arecibo (PALFA) but not as good as FAST, however it will have a significantly larger FoV and have multiple passes. Our LOTAAS (LOFAR) survey uses both coherent and incoherent beams and will make three passes with the incoherent beam. However it is at much lower frequency and so is highly complementary. None of these surveys can match MeerTRAP for the number of visits 
to the same regions of the sky (e.g. piggybacking on the MLSP pulsar timing project) or the total observation time.

As we are searching for a range of pulsar types that are currently under represented in our known sample because they are hard to detect with the usual pulsar survey approach, it is difficult to estimate how many new pulsars will be discovered. However, we have run simulations that show that MeerTRAP is extremely sensitive even in a single pass pulsar survey. We have determined that by piggybacking we will be able to sample the majority of the Galactic plane $\left(-120^{\circ}<1<30^{\circ}\right.$, $|\mathrm{b}|<15^{\circ}$ ) and we will find a few hundred new pulsars and up to 150 new MSPs. Piggybacking on the deep survey MLSPs gives us the sensitivity to measure the very important low luminosity limit for pulsars. If there were to be a program to undertake a large survey of the Large Magellanic Cloud (for example in Open time) that we could piggy back on our simulations show we could find at least 100 new pulsars and have the sensitivity and time resolution to find extragalactic MSPs.

\section{Conclusions}

MeerTRAP is a project to undertake fully commensal pulsar and fast transients searches in real time using MeerKAT. The combination of the sensitivity, field-of-view, large on sky time, and resolution of MeerKAT combined with the hardware and software implemented by the MeerTRAP and MPIfR teams will result in a system capable of discovering hundreds of fast radio bursts, and most importantly localising some of them to (sub)arcsecond positions. In combination with MeerLICHT this will provide a unique data set for understanding the transient radio sky at high time resolution. It will also be able to reveal hundreds of new pulsars and will be particularly sensitive to those which vary for any reason, be it some form of intermittency, binarity, scintillation or through evolution of the system.

We gratefully acknowledge the support of the MeerKAT and SKA SA teams and the MLSP PIs. MeerTRAP is a project that has received funding from the European Research Council (ERC) under the European Union's Horizon 2020 research and innovation programme (grant agreement No. 694745) and benefits from collaborations with the MPIfR and the University of Oxford.

\section{References}

[1] D. Thornton, et al., A Population of Fast Radio Bursts at Cosmological Distances, Science 341, 53 (July 2013).

[2] D. R. Lorimer, et al., A Bright Millisecond Radio Burst of Extragalactic Origin, Science 318, 777 (November 2007).

[3] D. J. Champion, et al., Five new fast radio bursts from the HTRU high-latitude survey at Parkes: first evidence for two-component bursts, MNRAS 460, L30 (July 2016).

[4] L. G. Spitler, et al., Fast Radio Burst Discovered in the Arecibo Pulsar ALFA Survey, ApJ 790, p. 101 (August 2014).

[5] K. Masui, et al., Dense magnetized plasma associated with a fast radio burst, Nature 528, p. 523 (December 2015).

[6] M. Caleb, et al., The first interferometric detections of fast radio bursts, MNRAS 468, 3746 (July 2017). 
[7] S. R. Kulkarni, E. O. Ofek, J. D. Neill, Z. Zheng and M. Juric, Giant Sparks at Cosmological Distances?, ApJ 797, p. 70 (December 2014).

[8] H. Falcke and L. Rezzolla, Fast radio bursts: the last sign of supramassive neutron stars, $A \& A \mathbf{5 6 2}$, p. A137 (February 2014).

[9] T. Totani, Cosmological Fast Radio Bursts from Binary Neutron Star Mergers, PASJ 65 (October 2013).

[10] J. M. Cordes and I. Wasserman, Supergiant pulses from extragalactic neutron stars, MNRAS 457, 232 (March 2016).

[11] L. G. Spitler, et al., A repeating fast radio burst, Nature 531, 202 (March 2016).

[12] E. Petroff, et al., A real-time fast radio burst: polarization detection and multiwavelength follow-up, MNRAS 447, 246 (February 2015).

[13] E. F. Keane, et al., The host galaxy of a fast radio burst, Nature 530, 453 (February 2016).

[14] R. Cen and J. P. Ostriker, Where are the baryons?, ApJ 514, 1 (1999).

[15] W. Deng and B. Zhang, Cosmological Implications of Fast Radio Burst/Gamma-Ray Burst Associations, ApJL 783, p. L35 (March 2014).

[16] M. McQuinn, Locating the "Missing" Baryons with Extragalactic Dispersion Measure Estimates, ApJ 780, p. L33 (January 2014).

[17] B. Zhou, X. Li, T. Wang, Y.-Z. Fan and D.-M. Wei, Fast radio bursts as a cosmic probe?, PhysRevD 89, p. 107303 (May 2014).

[18] B. Schutz, Determining the Hubble constant from gravitational wave observations, Nature 323, 310 (1986).

[19] N. Wex, Testing Relativistic Gravity with Radio Pulsars, ArXiv e-prints (February 2014).

[20] B. W. Stappers, et al., A State Change in the Missing Link Binary Pulsar System PSR J1023+0038, ApJ 790, p. 39 (July 2014).

[21] E. F. Keane and M. A. McLaughlin, Rotating radio transients, Bulletin of the Astronomical Society of India 39, 333 (September 2011).

[22] E. F. Keane and M. Kramer, On the birthrates of Galactic neutron stars, MNRAS 391, 2009 (2008).

[23] M. Kramer, A. G. Lyne, J. T. O’Brien, C. A. Jordan and D. R. Lorimer, A Periodically Active Pulsar Giving Insight into Magnetospheric Physics, Science 312, 549 (April 2006).

[24] M. Obrocka, B. Stappers and P. Wilkinson, Localising fast radio bursts and other transients using interferometric arrays, MNRAS 579, p. A69 (July 2015).

[25] R. J. Lyon, J. M. Brooke, J. D. Knowles and B. W. Stappers, A Study on Classification in Imbalanced and Partially-Labelled Data Streams, ArXiv e-prints (July 2013).

[26] R. J. Lyon, et al., Fifty Years of Pulsar Candidate Selection: From simple filters to a new principled real-time classification approach, MNRAS 459, 110 (2017). 